\title{
EL PROCESO DE RADICALIZACIÓN YIHADISTA DE MUJERES EN ESPAÑA
}

\author{
María Isabel García García \\ Instituto Gutiérrez Mellado, Universidad Nacional de Educación a Distancia \\ Title: The process of jihadist radicalization of women in Spain
}

Resumen: La radicalización violenta es un fenómeno complejo que debe ser explicado a través de un enfoque multidisciplinar. El objetivo de este artículo es explicar cómo y por qué se ha producido la radicalización violenta de 22 mujeres en España tras la irrupción de Daesh en el panorama internacional. Para logarlo, el estudio analiza los factores sociopolíticos que conducen a un individuo a abrazar la militancia islámica a través de tres niveles: macro, micro y meso. La investigación se sustenta en entrevistas a mujeres que han sido detenidas por integración en dicha organización terrorista, así como en un sumario y resoluciones de la Audiencia Nacional.

Palabras clave: radicalización, mujer, terrorismo, yihadismo

Abstract: Violent radicalization is a complex phenomenon that must be explained through a multidisciplinary approach. The aim of this article is to explain how 22 women in Spain became radicalised. To achieve this, the study analyses the socio-political factors that lead an individual to get involved with the Islamic militancy through three levels: macro, micro and meso. The research is supported by interviews with females in Spain who were convicted of terrorist offences and experts in the field; by judgement and indictments ordered by the Audiencia Nacional.

Keywords: radicalization, women, terrorism, jihadism

\section{Introducción}

Las mujeres han tenido presencia en diferentes grupos terroristas a lo largo de la historia. Sendero Luminoso, el IRA, las Brigadas Rojas, ETA o Los Tigres de Liberación de Eemal Tamil (LTTE, por si siglas en inglés) 
son solamente algunos ejemplos de las organizaciones que han contado con mujeres entre sus miembros. Sin embargo, la gran mayoría de las investigaciones sobre terrorismo han centrado su atención en descubrir cuáles eran las motivaciones de los hombres para unirse a causas violentas, asumiendo que el papel que desempeñaban las mujeres en estos grupos era secundario (Cunningham 2003; Taylor y Jacques 2008).

Existe una escasa literatura que haya abordado las causas que conducen a una mujer a comulgar con una doctrina extremista y la mayoría no vieron la luz hasta entrado el año 2000 (Speckhard 2008; Cragin y A. Daly 2009). La comunidad académica no se mostró unánime en cuanto a las causas y hubo dos tendencias que trataron de explicar el fenómeno. Así, una corriente sostiene que la principal razón atiende a la vertiente personal y que los vínculos familiares, la pérdida de un ser querido o haber sufrido una agresión sexual son los motivos más recurrentes para unirse a un grupo terrorista (Victor 2004; Bloom 2011). Otros autores apuntan a que esta postura solo perpetúa el estereotipo de mujer sumisa y víctima, negándoles la libertad de elección personal. Estos académicos sostienen que las mujeres abrazan la doctrina extremista por las mismas razones que los hombres y que la ideología o el nacionalismo son algunos de estos motivos (Cunningham 2003 y O’Rourke 2009).

La autoproclamación del califato por parte de Daesh en junio del año 2014 logró despertar el interés de la comunidad académica atraídos, en su mayor parte, por la gran movilización femenina (Peresin y Cervone 2015; Bakker y de Leede 2015). Se estima que de los 41.490 extranjeros occidentales que se han marchado a los territorios controlados por el grupo terrorista, alrededor de 4.162 son mujeres (Cook y Vale 2018, 14), una cifra única en un grupo terrorista. Una de las primeras y más importantes aportaciones científicas que trataron de explicar dicho fenómeno fue la de los académicos Hoyle, Bradford y Frenett en el año 2015. Los autores se adentran en las razones que las propias mujeres dirimen en sus redes sociales para unirse a la organización. Este trabajo tiene su continuidad meses más tarde en el desarrollado por Saltman y Smith (2015), quienes identifican una serie de factores de presión y atracción que hacen a las mujeres más predispuestas a apoyar a la organización.

Tabla 1: Factores de presión y atracción de acuerdo a Saltman y Smith

\begin{tabular}{|l|c|}
\hline Factores de presión & Factores de atracción \\
\hline - Sentimiento de exclusión social y cultural y & - Obligación religiosa \\
cuestionamiento de la identidad y pertenencia & de construir el Ca- \\
- Sentimiento de persecución por parte de la co- & lifato \\
munidad musulmana & - Sentimiento de \\
- Enfado, tristeza y frustración sobre la actua- & pertenencia y her- \\
ción de la comunidad internacional ante la per- & mandad \\
secución percibida & - Aventura romántica \\
\hline
\end{tabular}


Según señalan los autores, los factores de presión presentes en los individuos los hacen más susceptibles a la propaganda extremista ya que el grupo logra canalizar estos sentimientos presentes en las mujeres para hacerlas comulgar con la causa a través de los factores de atracción. Estos son los que empujan a los individuos mediante incentivos positivos, como el otorgamiento de una función que desempeñar dentro del califato, hacia el extremismo islamista (Saltman y Smith, 2015). Por tanto, este estudio apuntaba que estos factores no pueden ser entendidos de manera aislada, sino que es necesaria una interacción entre algunos de ellos para que finalmente se produzca la vinculación a Daesh. Asimismo, estas incipientes investigaciones también adelantaban que no existe un único perfil entre las mujeres que se habían unido al grupo yihadista.

Desde entonces una gran parte de los estudios dedicados a analizar las causas que hay detrás de la movilización femenina lo han hecho a través de la propaganda difundida por el grupo a través de la red (Rafik y Malik 2015; Tarras-Wahlberg 2016; Lahoud 2018).Y es que a pesar de los esfuerzos por entender el fenómeno del terrorismo se trata de una de las áreas con menos rigor científico del mundo académico (Schmid y Jongman, 2005) debido, en parte, a la gran dificultad de acceso a datos empíricos. En los últimos años y gracias a las ventajas operativas que aportan las nuevas tecnologías, la comunidad científica ha intentado suplir la falta de fuentes primarias en este campo utilizando las redes sociales. Se trata de una herramienta al alcance de todos y muy utilizada por los individuos que se adhirieron a la causa de Daesh por lo que no es de extrañar que una parte de estos pioneros estudios basaran sus conclusiones en esta fuente de información.

Pese al repunte que han experimentado los estudios que buscan explicar las causas que llevan a una mujer a asumir prácticas violentas, lo cierto es que aún existe un gran desconocimiento acerca del fenómeno (Sjoberg, 2018). Esto es debido a que la mayoría de los estudios se han focalizado casi exclusivamente en la descripción del suceso y en analizar las causas que llevan a las mujeres a apoyar a Daesh dejando de lado el estudio de los procesos psicosociales que hacen que un individuo no violento inicie un proceso de radicalización y acabe convirtiéndose en un terrorista (Davis 2017, 32).

El presente artículo cubre las carencias que se han descubierto tras el repaso a los principales estudios sobre la materia, respondiendo a por qué algunas mujeres han comenzado a cometer delitos relacionados con el terrorismo yihadista en España. El objetivo del estudio es aportar luz sobre esta cuestión y dilucidar cómo han llegado algunas mujeres residentes en el país a experimentar un proceso de radicalización que les ha llevado a apoyar a Daesh. 


\section{Método}

Para comprender qué ha llevado a mujeres españolas a cometer delitos relacionados con el terrorismo es imprescindible aclarar que se entiende por proceso de radicalización violenta. Este se podría definir como «un proceso dinámico que conduce a la persona a una visión extrema y dicotómica de la realidad, donde se acepta y promueve el uso de la violencia como medida para conseguir instrumentalmente los objetivos del grupo o movimiento» (Trujillo et al. 2018, 4). Diversos investigadores (Horgan 2005; de la Corte 2006; McCauley y Moskalenko 2011) han tratado de ofrecer respuestas a dicho proceso desde una perspectiva psicosocial, asumiendo que el fenómeno no puede explicarse con variables sociales o psicológicas, sino que es necesaria una interacción entre ellas. Este estudio se enmarca en dicho enfoque multidisciplinar para comprobar si se pueden constatar en el caso de mujeres radicalizadas en España.

La base documental en la que se apoya el artículo para responder a su pregunta de investigación se extrae de una muestra de 22 mujeres que han sido procesadas por la Audiencia Nacional por delitos de terrorismo o por las que existe una orden de detención por haberse integrado en las filas del grupo tras desplazarse a Siria o Irak. Teniendo en cuenta que a las Fuerzas de Seguridad del Estado les consta que 26 mujeres con nacionalidad española y/o residencia en el país se han integrado en las filas de la organización en Siria o Irak ${ }^{1}$, se trata de una muestra bastante representativa para poder extraer inferencias válidas sobre el fenómeno. Más aún si se tiene en cuenta que no existe un estudio que aglutine tantos casos para explicar el proceso de radicalización por el que atravesaron varias mujeres hasta verse atraídas por la doctrina de Daesh.

La muestra se empezó a configurar en el año 2014 y a partir de la primera operación policial en la que una mujer fue detenida por delitos relacionados con el terrorismo yihadista en España. Las 22 mujeres se seleccionaron por su implicación en dicha causa, así como por las distintas operaciones que se abrieron a raíz de esta primera investigación. De esta manera, los documentos de información que se manejan para elaborar dicho perfil corresponden al sumario 05/2016 de fecha 2 de febrero de 2016 del Juzgado de Instrucción $n^{\circ} 1$ de la Audiencia Nacional, sobre los atestados de la primera investigación policial conocida como 'Operación Kibera' así como otras resoluciones del mismo órgano judicial.

Además, se han llevado a cabo tres entrevistas personales a jóvenes procesadas por la Audiencia Nacional. Dos de estas mujeres fueron condenadas por dicho órgano a cuatro años de prisión por integración en organización terrorista en el marco de la Operación Kibera. El Tribunal

1 Datos proporcionados por la Unidad Central Especial número 2 (UCE-2) con fecha 5 de diciembre de 2018. 
Supremo ha ratificado la condena de siete años de la tercera, también por integración en organización terrorista. Los encuentros se produjeron durante el mes de septiembre de 2018. Uno de ellos fue en la ciudad de Ceuta, donde se tuvo la oportunidad de hablar durante dos días con una de las jóvenes. Las entrevistas con las otras dos mujeres se sucedieron en Madrid también durante el mes de septiembre de 2018. Se registraron en total dos encuentros, en los que se tuvo la ocasión de hablar en solitario con cada una de ellas y se llevaron a cabo entrevistas semiestructuradas. Estas pidieron no ser identificadas a lo largo del texto debido al estigma social que les ha supuesto su implicación con el grupo por lo que se utilizarán sus iniciales cuando se introduzcan fragmentos de sus respuestas.

El artículo se divide en tres niveles de análisis: macro, micro y meso. El primero atiende a los factores relacionados con la coyuntura internacional, así como el entorno socioeconómico y cultural del que proviene el individuo, el segundo se centra en la persona y el tercero en sus relaciones sociales.

\section{Análisis nivel macro}

A lo largo de la historia han existido diferentes movilizaciones transnacionales de índole yihadista. Los conflictos de Irak o Somalia, por ejemplo, consiguieron atraer a miles de combatientes de diversas partes del mundo. Y es que determinados hechos pueden iniciar un fenómeno y actúan como precondicionantes (Jordán 2009, 201). Por eso, el proceso de radicalización violenta que han experimentado varias mujeres en España no puede ser entendido sin enmarcarlo dentro del contexto internacional. Así, el establecimiento del califato en junio de 2014 por parte de Abu Bakr al Baghdadi en Mosul tuvo un gran efecto movilizador que repercutió en el ámbito local de la mayoría de los países occidentales. Se trataba de un gesto con una gran carga simbólica, ya que el último califato fue el Otomano, abolido en 1924. En el discurso de esta autoproclamación Abu Bakr mandó un mensaje incitando a todos los musulmanes, mujeres y hombres, a abandonar sus países de residencia e iniciar la migración para vivir bajo el que Daesh consideraba el único estado verdaderamente islámico (Moubayed 2016, 244).

Coincidiendo con la fecha de autoproclamación del Califato y entre los años 2014 y 2015 se produce la movilización de las mujeres de la muestra. De hecho, es en agosto de 2014 cuando se produce la primera detención de una mujer por delitos relacionados con el terrorismo yihadista en territorio español (García-Calvo 2017). El 59 por ciento fueron detenidas, intentaron unirse o se unieron a la organización durante el primer año. El 40,9 por ciento restante fueron detenidas durante el año 2015 por delitos relacionados con el terrorismo yihadista. 
La coyuntura internacional no es el único indicador que hay que tener en cuenta en este nivel de análisis, la interpretación que se hace sobre un determinado acontecimiento y la manera en la que se traslada a la audiencia son factores que hay que tener en cuenta. En este caso, la guerra de Siria sirvió a Daesh para señalar que la población musulmana estaba siendo víctima de un sufrimiento extremo y que el culpable de dicho padecimiento era la comunidad internacional, así como el régimen de Bashar al Asad. Este marco de injusticia, que configura y condensa el mundo, logra cohesionar el movimiento potenciando su adhesión a una causa (Snow y Benford 1992, 137). En este caso, tomar partido por la víctima de la injusticia social.

Internet fue el canal elegido para trasladar el mensaje y las plataformas más utilizadas fueron Twitter, Facebook, Telegram o WhatsApp. El 90\% de las mujeres de la muestra tuvieron acceso a este contenido a través de estas aplicaciones, bien por la participación directa en la divulgación de estos mensajes, como por la recepción de ellos tras ser miembros de diferentes grupos. Por ejemplo, una de las mujeres procesadas por la Audiencia Nacional publica en 2014 en su perfil de Facebook el siguiente estado: ‘ ¿Cómo pueden seguir apoyando a Occidente...Mira lo que hacen ellos con nuestros hermanos. ¿Y nosotros somos los terroristas?’ Con lo que dejaba claro que hay dos bandos y había que tomar partido (Audiencia Nacional [AN], Escrito de acusación del fiscal, sumario 5/2016, 49).

La elección de estas plataformas no es casual. Los medios de comunicación de masas están aumentando las ventajas estratégicas en el activismo internacional (Bennett 2003) y Daesh ha sabido utilizarlo en su beneficio para reducir costes y facilitar su proyección. Por primera vez un grupo terrorista era capaz de controlar el mensaje y de estar en contacto con su audiencia sin necesidad de intermediarios. De esta manera, desde Siria, Irak o el no tan lejano Marruecos, miembros del grupo podían comunicarse con mujeres para transmitir su ideario. El 68\% de las mujeres de la muestra mantenía contacto con algún miembro de la organización criminal a través de las redes sociales fuera del territorio nacional. Sin embargo, solamente ocho mujeres de la muestra iniciaron su proceso de radicalización puramente online y sin tener ningún estímulo offline. Esta cifra indica que si bien es un medio eficaz para transmitir la ideología yihadista no ha sido, al menos en el caso español, el principal medio de captación.

En relación al entorno social y económico de las mujeres de la muestra, investigaciones recientes (Gustafsson y Ranstorp, 2017) señalan que la gran mayoría de los individuos radicalizados en Occidente provienen de zonas desfavorecidas. Cerca del $56,5 \%$ de las mujeres de la muestra proviene de barrios españoles considerados vulnerables ${ }^{2}$ o tienen raíces

2 En términos urbanos la vulnerabilidad se referiría a la posibilidad de que la población de un determinado espacio concreto sea afectada por circunstancias adversas, de 
en esas zonas. Las barriadas de El Príncipe Alfonso en Ceuta y La Cañada de Hidum en Melilla se sitúan como los lugares de residencia de la mayoría de las mujeres de la muestra, un total de $43 \%$, por lo que se considera necesario analizar las características de dicho enclave.

La Fiscalía General del Estado señalaba en el año 2016 que dos de los principales focos de la actividad yihadista en España eran Ceuta y Melilla (Memoria 2016, 276). Se trata de enclaves situados en el norte de África y fronterizos con Marruecos. Su proximidad ya supone un riesgo para las ciudades españolas ya que es uno de los países con más mujeres migrantes a la causa de Daesh (Cook y Vale, 2018, 22). Las dos ciudades han tenido episodios migratorios de población musulmana del contiguo Marruecos y gracias a su cercanía y por motivos laborales aún se mantienen vínculos culturales y familiares muy estrechos (Rontomé 2015, 2). Ceuta y Melilla representan la mayor concentración de población musulmana de España, con un 43\% y 52\% respectivamente (Unión de Comunidades Islámicas de España [UCIDE] 2018) y la mayoría son ciudadanos españoles (UCIDE 2018). Gracias a elevadas tasas de natalidad, estos colectivos integran a esa segunda y tercera generación descendientes de los primeros migrantes (De la Corte 2015, 11). Son los individuos que pertenecen a esta generación los más vulnerables a sufrir un proceso de radicalización violenta ya que experimentan dificultades a la hora de definirse a ellos mismos dentro de la sociedad en la que viven. Esas dificultades son más acusadas durante la adolescencia (Saltman y Smith 2015, 9). El 54\% de las mujeres de la muestra no superaban los 23 años de edad en el momento de los hechos.

Asimismo, estas dos ciudades tienen una de las tasas más alta de desempleo de España y es la población joven la que sufre el mayor impacto. En Melilla, por ejemplo, un 62.7\% de los desempleados son personas de entre 15 y 24 años (Eurostat 2017). Además, en Ceuta la exclusión social y la pobreza afecta a un a un $65 \%$ de la población musulmana y solo a un $15 \%$ de la población de origen cristiano y otras minorías (Rontomé 2015, 3). En relación a la Educación, la tasa de abandono escolar se situaba en 2017 entorno al 20\% en Ceuta y en el 27\% en Melilla, por encima de la media española que se encuentra en el 18,3\% (Instituto Nacional de Estadística [INE], 2017). Dichos datos afectan en mayor medida a la población musulmana. En Ceuta, por ejemplo, la tasa de fracaso escolar de los niños musulmanes alcanza el 70\% (Rontomé 2015, 2). En el caso de las mujeres, la tasa de abandono escolar en Ceuta es la más alta de toda España, situándose en el 24, 4\%, y se aleja casi 10 puntos de la media del país $(15.1 \%$,$) . En el resto de las comunidades autónomas son los hom-$

modo que el concepto alude no tanto a la existencia de una situación crítica constatada en la actualidad como a la presencia de unas determinadas condiciones de riesgo, fragilidad y desventaja que harían posible la entrada en esa situación crítica de desfavorecimiento (Hernández et al, 2018, 15). 
bres los que dejan antes los estudios (A.Q. 2018). Estos datos, por tanto, suponen un caldo de cultivo para entender cómo se acaba forjando el proceso de radicalización.

\section{Análisis nivel micro}

Existen una serie de factores presentes en los individuos que aumenta la vulnerabilidad de las personas a sufrir un proceso de radicalización violenta (Torres-Marín et al. 2017, 138). Con la base documental a la que se ha tenido acceso, se ha podido identificar algunos de estos factores, entre los que se encuentra la presencia de estados de crisis personal y de pérdida de autonomía e integridad psicológica, déficit de apoyo familiar, escasa cualificación académica o procedencia de entornos marginales.

Se ha comprobado que 20 mujeres de la muestra estaban atravesando un mal momento personal cuando empezaron a verse atraídas hacia el extremismo. Las propias jóvenes a las que se ha entrevistado relacionan atravesar un mal momento con verse atraídas hacia el extremismo. Entre los episodios que enumeran y que se han identificado, están la muerte de un ser querido, una ruptura sentimental, punto de inflexión tras sufrir un accidente de tráfico o problemas de comportamiento en el centro de estudio (AN, sumario 5/2016, tomo 1, 88). Asimismo, en la base documental analizada también se describen estados de ánimos de crisis personal. Dos mujeres apuntaban en una conversación que «se encontraban perdidas» antes de iniciar el proceso y que su adhesión al grupo les dio sentido a su vida (AN, sumario 5/2016, tomo 21, 6555)

Una de las jóvenes entrevistadas reconocía que no estaba anímicamente bien. Tras cumplir 18 años esperaba que su padre, al que no veía desde pequeña, fuera a buscarla. Su déficit de apoyo familiar y una personalidad de baja robustez psicológica la hicieron más vulnerable a las presiones del grupo de amigas ${ }^{3}$. Esta falta (o percepción) de apoyo familiar antes de iniciar el proceso de radicalización también se ha visto en otras dos jóvenes. Una de ellas, que provenía de una familia evangelista de origen latinoamericano, se crio en un ambiente estricto en el que los roles de género estaban muy marcados. Desde los 13 años comenzó a fugarse de su domicilio y a consumir alcohol, situación por la que llegaron a intervenir los servicios de protección de menores y llegó a tratar de llamar la atención de su familia autolesionándose. Tras unos años viviendo en España, la joven señala que su familia decide volver a su país de origen debido a la crisis, pero que no quieren que regrese con ellos. El único arraigo familiar que le quedaba entonces en España, además de un hijo de corta edad, era una tía con la que estuvo viviendo una temporada.

\footnotetext{
3 En el siguiente apartado se explica la forma en la que funcionaba el grupo.
} 
Una evaluación psicológica a la que fue sometida dictaminó que se trataba de «una persona controlada, con tendencia a la escasez de emociones positivas que revelaría un carácter pesimista, socialmente introvertida y centrada en sí misma». El informe también señalaba que poseía «un estilo de pensamiento constructivo de alto afrontamiento personal, autoaceptación, autoestima y sin presencia de rumiaciones» (AN, sumario 5/2016, tomo 21, 7043). La ex pareja de esta mujer, un joven marroquí, afirmaba que la joven hacía todo lo que él decía y que una amiga suya, también procesada por la Audiencia Nacional por integración en organización terrorista, era igual de fácil de convencer (AN, sumario 5/2016, tomo 8, 2068-2070). Algo que también confirma una prima de la joven en una entrevista (TV3 2015).

La sumisión al hombre se ve en varios de los perfiles de la muestra. Una de las mujeres entrevistadas acababa de salir de una relación con una pareja que describe como dominante y con la que había estado alrededor de tres años. Controlaba sus movimientos, incluso viviendo fuera de la ciudad en la que residía la mujer. «Mi familia me presionaba para que dejara esa relación y tras esta ruptura buscaba algo espiritual en mi vida» (Entrevista a C. B. Septiembre de 2018. Ceuta). Esta misma joven también había sufrido brotes de depresión durante su adolescencia y había acudido al psiquiatra para tratarse (Entrevista al abogado Álvaro Sena. Septiembre de 2018. Madrid). Otra de las mujeres de la muestra señala en varias conversaciones que mantiene con otro de los detenidos durante la Operación Kibera y con el que mantenía una relación sentimental, que «necesita un marido que la enseñe ya que ella no sabe casi nada de religión» (AN, Sumario 5/2016, tomo 3, 814). Con esa frase asume que ella no es capaz de ser autónoma a la hora de aprender y que esta educación depende exclusivamente del hombre. No obstante, esta llega a acusarle en una ocasión de machista por su comportamiento y le pregunta si entre ellos va a haber igualdad. Él cierra el posible debate señalando que «el hombre está un grado por encima de la mujer ya que así lo ha querido Alá y que su función es obedecer al marido» (AN, sumario 5/2016, tomo 4, 1026). A otra de las mujeres de la muestra le imponen que para casarse con ella debe usar burka, parir muchos hijos, obedecer al marido y no tener vida social (AN, sumario 33/2018, 57), dicha justificación la atribuye a la religión.

Es durante la etapa educativa cuando los individuos adquieren parte de las habilidades necesarias para poder hacer frente a estos discursos extremistas por lo que el nivel educativo posee cierta importancia en este fenómeno (Torres-Marín et al. 2017, 138). Se ha podido comprobar que el $50 \%$ de las mujeres de la muestra se encontraba matriculada realizando algún tipo de estudio. El 47,6 \% no estaba en posesión del graduado escolar aunque una aun no tenía edad de tenerlo y se encontraba matriculada en el instituto. Una de ellas acudía a un curso de Formación Profesional en Melilla gracias al que ingresaba 400 euros mensuales (AN sumario 5/2016, Tomo 1, 163) y dos se encontraban estudiando un Grado 
Medio y de Técnico Superior. Tres mujeres tenían estudios superiores, solamente una de ellas estaba realizando una carrera.

Varias, además, mostraban su pesimismo a la hora de encontrar trabajo (AN, sumario 5/2016, tomo 3, 806) y una, pese a estar formándose en un curso de Técnico Superior, también señalaba que en «España nos están matando con tanta crisis» (AN, Auto de procesamiento, 3/2016, 32). Esta incertidumbre laboral puede generar sentimientos de frustración que los grupos yihadistas logran canalizar. Casi el 48\% de las mujeres no desempeñaba ninguna actividad en el momento de ser captadas y provenían de un ambiente marginal. Una de las mujeres, por ejemplo, había sufrido un desahucio, tres cobraban una prestación económica del Estado (AN, sumario 5/2016, tomo 1,163 , tomo 6,1328 , tomo 13,4158 ), otra se encontraba, según una amiga, en una situación de semi-abandono (AN, sumario $5 / 2016$, tomo 8,2049 ) y una de las menores también venía de una familia con una situación precaria (AN, sumario 5/2016, tomo 2, 416).

Los entornos de marginación social (real o percibida) también son indicadores de vulnerabilidad para los individuos y sufrirlos aumenta las probabilidades de asumir un comportamiento extremista violento (Pretus et al. 2018). Las personas pueden considerar que son objeto de una injusticia social por tener ciertas creencias o pertenecer a determinado estatus social (Trujillo 2009.). Las mujeres entrevistadas señalaban que habían sido objeto de burla por llevar pañuelo y que no tenían las mismas oportunidades que las personas de origen cristiano a la hora de, por ejemplo, encontrar un trabajo. Este sentimiento de marginalización social también puede derivar en una desconfianza hacia las instituciones. Por ejemplo, tras la desaparición de una de las jóvenes de su casa, su familia tardó dos días en acudir a la policía a presentar la denuncia; otras no han acudido nunca a las Fuerzas de Seguridad del Estado e incluso han traído a sus hijas de vuelta de Siria a través de intermediarios (AN, sumario 5/2016, tomo 9, 2577).

El escaso conocimiento del Islam también es una tónica entre las mujeres que son objeto de análisis y así lo confirman agentes que han investigado varias de estas operaciones (Entrevista policías del Departamento de Comunicación de la Comisaría General de Información de la Policía Nacional [CGI]. Noviembre de 2018. Madrid). Lo recogen los hechos recogidos en el sumario 5/2016 de la AN y las propias jóvenes que han sido entrevistadas. Las tres, pese a venir de familias tradicionalmente musulmanas, señalan que no eran muy practicantes y su conocimiento del Islam era escaso en el momento de comenzar el proceso de radicalización. Una de ellas apunta que empezó a verse envuelta en el extremismo después de mostrar interés en aprender más de la religión. "Quería ser mejor persona y el Islam tiene unos valores en los que quería verme reflejada, llegué a creerme lo que me contaban, en parte, por no conocer mi religión» (Entrevista a S.B. septiembre de 2018. Madrid) 
Estos factores que se han encontrado en las mujeres de la muestra no tienen por sí solos que desembocar en un proceso de radicalización violenta. Su presencia, no obstante es aprovechada por los miembros del grupo para hacer calar su doctrina.

\section{Análisis nivel meso}

Este nivel de análisis comprende los factores que conciernen a las redes sociales del individuo, ya que las relaciones de amistad o de parentesco son de crucial importancia para entender el cambio que está detrás de un determinado comportamiento social (Sageman 2004,109-113). Las personas con las que nos relacionamos ejercen influencia sobre nosotros y «facilitan la socialización en determinados valores» (Jordán 2009, 208) sean o no extremistas. Se ha podido comprobar que el 59\% tenía un vínculo emocional con alguien que, o bien había sido procesado por delitos relacionados con terrorismo, o se había trasladado a Siria. En un 40\% de los casos se trataba de un familiar. Dos mujeres, por ejemplo, entraron en contacto con el grupo tras preguntar por el destino de un familiar que se había desplazado a Siria (Saíz-Pardo, 2016).

La influencia ejercida por las parejas sentimentales también puede convertirse en la principal fuente para entrar en contacto con la ideología extremista. Para el 22,7\% de los casos la relación de parentesco consistía en la de una pareja sentimental. Una joven melillense pareja de un marroquí que se dedicaba a captar a jóvenes en la Cañada de Hidum (AN, Auto de procesamiento, 3/2016,) fue detenida tras haber abandonado territorio sirio junto a su hija menor de edad. Una amiga de la joven señala que su cambio se produjo por influencia de un marido dominante, ya que antes no comulgaba con ideas extremistas. Otras dos mujeres se convirtieron al Islam tras conocer a los que serían sus maridos y acabaron asumiendo la doctrina yihadista con la que ellos simpatizaban. Uno cumplía condena en Marruecos por terrorismo cuando su mujer abandonó España junto a sus hijos para reunirse con parte de su familia política en Siria (Escrivá, 2018).

Las relaciones de amistad también han sido un factor clave en este engranaje. Según estableció el académico Maslow (1943), los individuos, una vez cubiertas sus necesidades básicas, experimentan una necesidad de pertenecer a un grupo social con el que identificarse. No lograr formar parte de uno puede conducir a sentimientos de frustración o baja autoestima que los hace más vulnerables a buscar esa aceptación. Gran parte de las mujeres de la muestra se sentían solas y no tenían apenas amigos cuando comenzaron a adquirir una visión radical de la religión. Este fue el caso de una mujer originaria de Cádiz, proveniente de una familia desestructurada y que durante su infancia y adolescencia sufrió episodios de discriminación por su obesidad. Cuando se convirtió al 
Islam encontró una comunidad que satisfizo su deseo de ser finalmente aceptada (Entrevista a policías de CGI. Noviembre de 2018. Madrid). «Los grupos de amigos pueden conducir al radicalismo de dos maneras: porque desde un principio tienen esa finalidad (que sean promovidos por uno o varios yihadistas) o porque dentro de un grupo preestablecido, uno o varios de sus miembros se acercan al yihadismo y arrastran a los demás» (Jordán 2009, 209).

Parte de las mujeres, un $45 \%$, se conocían previamente y pertenecía a los mismos círculos sociales. Este fue el caso de una joven menor de edad radicalizada por la influencia de su pareja ${ }^{4}$ y que influyó en varias mujeres de su entorno, como su cuñada, quien una vez asumida la visión radical también intentó adoctrinar a varias amigas. Estas dos jóvenes ejercían una especie de liderazgo sobre la conducta del resto de chicas de su entorno, que en algunos casos pasaron de usar ropa occidental a niqab en tan solo siete meses (AN, sumario 5/2016, tomo 4, 1194). Sin embargo, muchas afianzaron lazos después de coincidir en diferentes grupos creados en las redes sociales. Se ha podido comprobar que el $68 \%$ coincidieron alguna vez o tuvieron contacto entre sí a través de grupos en Facebook o WhatsApp en alguna ocasión durante el tiempo que duró su proceso de radicalización.

La principal herramienta de comunicación entre ellas era a través de estas aplicaciones, pese a que algunos encuentros se producían en domicilios privados. Las redes sociales no se limitan solo a transmitir información, sino que son capaces de construir patrones culturales y crear una serie de contextos con los que los individuos se sienten a gusto y se identifican (Requena 2008). El hecho de que muchas mujeres se vieran afectadas por las mismas circunstancias o les atrajeran los mismos temas facilitó esta identificación compartida y esa transmisión de pautas de comportamiento al coincidir en estos grupos. Las tres mujeres entrevistadas señalan que en los grupos había bastantes participantes, generalmente estaban formados por mujeres y se trataban temas sobre religión o cocina, aunque era recurrente que alguien hiciera comentarios sobre Daesh. Posteriormente, cuando se empezaban a forjar lazos de afinidad entre las participantes y se mostraba cierto interés por la organización, se creaban grupos más pequeños de alrededor de 10 individuos, administrados casi siempre por una mujer (AN, escrito de acusación del fiscal, $5 / 2015,5-7)$.

Una vez que las jóvenes iban asumiendo la doctrina yihadista creaban sus propios grupos, por lo que el espacio de influencia crecía. Estos procesos doctrinales no respondían a una estructura jerárquica propia, ni funcionaban de manera horizontal únicamente CGI (Entrevista a po-

4 Fue detenido por pertenencia a organización terrorista y además se era el hermano de otra de las mujeres detenidas en la misma operación que la menor Ceutí. 
licías de CGI. Noviembre 2018. Madrid). No dominaban las técnicas de persuasión y funcionaban más de manera impulsiva a base de prueba y error (Entrevista al académico Humberto Trujillo. Febrero de 2019. Madrid). Era común durante este proceso que miembros del grupo se pusieran en contacto a través de las redes sociales con ellas para tratar de iniciar una relación sentimental o convencerlas para marcharse a Siria e Irak. Las propias amigas llegaban a pasar sus contactos y alguna actuó de facilitadora para miembros del grupo asentados en Marruecos. Dos jóvenes de Melilla llegaron a ofrecer sus casas para que las chicas captadas pernoctaran antes de cruzar la frontera del país vecino y poder coger un avión rumbo a los territorios controlados por Daesh (AN, 5/2016, tomo $6,1337$ y 1416$)$

\subsection{Proceso de adoctrinamiento}

Una vez descritas las vulnerabilidades que presentaban las mujeres de la muestra antes de ser captadas y las relaciones que tenían, se describirán los medios que se emplearon para su adoctrinamiento y cómo fue el proceso que atravesaron para reformar su pensamiento y llevarles a asumir la doctrina del grupo. Una de las principales técnicas de manipulación psicológica que conducen a una pérdida de autonomía del individuo consiste en aislar a los miembros de su soporte social habitual (Trujillo et al. 2018, 17). Se trata de una técnica que se ha identificado en varias de las mujeres de la muestra con las que se ha podido hablar. Sin embargo, no se hace por medios coercitivos sino que las mujeres voluntariamente llegan a romper esta relación con sus seres queridos cuando estos las increpan por su nueva visión rigorista de la religión. Una de las jóvenes menores de edad llevaba, por ejemplo, un año sin hablarse con su familia. Afirmaba que le era «difícil ser musulmana ya que su familia estaba en contra» (AN, sumario 5/2016, tomo 6,1359). Sin embargo, su familia sí que era practicante, pero no compartía la nueva visión de la joven.

El grupo aprovecha este momento por el que atraviesan las mujeres para presentar a sus familiares como infieles. "Me decían que no eran buenos musulmanes, ya que no practicaban la religión tal y como dicta el grupo y que no me entendían» (Entrevista. Septiembre de 2018. Madrid). A una de las jóvenes, que se había 'casado' con un miembro del grupo a través de la red ${ }^{5}$, su recién estrenado marido le pedía que dejara de escuchar a su tía y se reuniera con él de una vez en Siria (AN, sumario

5 La joven contrajo matrimonio con un miembro de Daesh de origen francés y que se encontraba en Siria en el momento en el que entraron en contacto. La joven llegó a grabar un audio de la ceremonia en la que participaron cuatro hombres, un Imán, dos testigos y el joven francés. Este tipo de enlaces, aunque no tenían validez, eran característicos durante el tiempo que estuvo vigente el Califato (AN, sumario 5/2016,1870). 
5/2016, tomo 16, 4848). Otra de ellas afirma que le aconsejaron apagar el teléfono y no despedirse de nadie cuando fuera a iniciar la migración. Esta, sin embargo, no hizo caso y confiesa que acabó dando marcha atrás por petición de su madre (Entrevista. Septiembre de 2018. Madrid).

Los reclutadores se designan como la verdadera familia y los únicos que de verdad velan por su bienestar. "Te acabas metiendo en un túnel en el que no ves más allá y solamente te relacionas con gente que piensa de la misma manera que tú, así que asumes que tienen razón» (Entrevista. Septiembre de 2018. Madrid). Este proceso refuerza los elementos identitarios de los individuos (Jordán 2009, 213) y a la vez deriva en lo que se conoce como control internalizado, «donde se percibe que la verdad es una posesión exclusiva del grupo» (Trujillo et al. 2018,13). Esta pertenencia y la búsqueda de determinada información por Internet también condujeron a que las mujeres sufrieran el llamado filtro burbuja. El interés de los individuos por ciertos temas en Internet puede conducir a una endogamia, ya que los algoritmos que utilizan las redes sociales acaban recomendando solo información relacionada con sus temas de interés. De esta manera, la esfera virtual también monopolizó sus opiniones.

Al final acaban generando una dependencia del grupo cada vez mayor que se acentúa al estar siempre conectadas a las redes sociales. Una de las jóvenes, por ejemplo, tenía un comportamiento obsesivo con el móvil y siempre lo tenía en la mano (AN, Auto de procesamiento, sumario $3 / 2016,49$ ). Casi todas contaban con varios terminales ya que vivían en constante temor de ser vigiladas por las Fuerzas de Seguridad del Estado (AN, Sumario 5/2016, tomo 6,1314; ANS, 33/2018,12). Esta dependencia se ha percibido incluso después de haber sido detenidas. Una de las jóvenes consultó en el grupo de amigas que conoció cuando comenzó a radicalizarse la posibilidad de llevar a cabo la entrevista con la autora de esta investigación. Una vez que ella accedió tres más tuvieron la predisposición a hablar de la situación por la que habían pasado.

El lenguaje que utilizan está cargado de refuerzos positivos y son íntimas, tanto es así que se interpelan utilizando el término ukty (mi hermana en árabe) y tratan de ayudarse, incluso ofreciéndose dinero para iniciar la migración pese a apenas conocerse. «Se interesan por tu vida y te lo pintan todo de colores» (Entrevista. Septiembre de 2018. Madrid). A la vez, las personas que ejercen de líderes dentro de los grupos también envían mensajes que desarrollan un sentimiento de antagonismo con el mundo exterior. Una de las jóvenes menores de edad señala en su declaración que en el grupo 'Muyahidines los guerreros de Alá' decían que "cuantos más seamos mejor, antes de que nos maten a nosotros» (AN, sumario $5 / 2016$, tomo 1,194 ) o que 'no podían quedarse quietas ante el atropello que sufren sus hermanos musulmanes' (AN, sumario 5/2016, tomo 6,1338). Por lo que se instauran bandos que suponen enfrentados.

Finalmente, algunas de las jóvenes señalan que llegaban a un punto en el que se sentían obligadas a desplazarse a los territorios controlados 
por la organización C. B (Entrevista. Septiembre de 2018. Ceuta). Después de haber asumido esta doctrina, la migración era la única vía posible para ser coherentes con ellas mismas.

No obstante, también se ha recurrido al miedo como estrategia de control y han sido frecuentes las presiones y las amenazas por parte de miembros del grupo cuando las mujeres han dudado acerca de desplazarse a Siria o Irak. Una joven llegó a denunciar que un individuo «se estaba poniendo en contacto con ella para proponerle de forma intimidatoria ir a Siria». A la joven le ofrecieron pagarle el viaje además de las deudas que tuviera contraídas (AN, sumario 5/2016, tomo 3, 934).

No es la única que hace alusión a estas presiones. Otra de las jóvenes menores de edad también se arrepintió cuando le expusieron que tendría que combatir en la zona de conflicto. Al negarse a realizar el viaje, le dijeron que vendrían a por ella si no cogía el avión. Las amenazas con acudir a la policía también estaban presentes. «Acabas sintiéndote como si estuvieras en una caja y que solo tienes dos opciones, o ir a la cárcel o marcharte» (Entrevista. Septiembre de 2018. Madrid).

\section{Conclusiones}

Tras el análisis se puede concluir que todas las mujeres de la muestra presentaban una serie de vulnerabilidades que las hicieron más predispuestas a escuchar la propaganda yihadista y a sufrir un proceso de radicalización violenta. En el primer nivel de análisis resulta fundamental el establecimiento del califato y el llamamiento a los musulmanes para que se produjera una movilización de tal envergadura. El mensaje que se trasladó sobre la opresión a la que estaba siendo sometida la comunidad musulmana repercutió a escala local y ayudó a justificar las acciones del grupo. Este tipo de discursos ofrecen un esquema interpretativo simple de lo que está sucediendo y obliga a los individuos a tomar partido por un bando con el que los individuos empatizan debido, generalmente, a la percepción que tienen sobre el trato que reciben en su lugar de residencia por el mero hecho de profesar una determinada religión.

El estado anímico por el que atravesaban las mujeres y su robustez psicológica parecen ser la clave que está detrás de las razones que les llevaron a iniciar ese proceso de radicalización. Si bien es necesario seguir profundizando en este nivel de análisis, ese punto de inflexión que se ha encontrado en gran parte de las mujeres de la muestra las sitúa en un momento idóneo para adaptar su pensamiento. Este, además, se ve reforzado por la presencia de otra serie de vulnerabilidades.

Pese a que la educación proporciona determinadas herramientas que ayudan a crear un pensamiento constructivo y a desactivar el mensaje del grupo, lo cierto es que parece tener más relevancia la marginación social 
y la idea de que debido a profesar la religión musulmana se está relegado a ser ciudadano de segunda clase. Esta percepción unida a una pérdida de sentido de la vida o a la crisis personal por la que atraviesan, acaba inclinando la balanza por una alternativa en la que emigrar significa tomar partido por alguien que se interesa por ellas y a un lugar donde creen que son valoradas. El papel que juega el entorno también se ha demostrado de vital importancia. La posterior influencia ejercida por el grupo y los efectos que puede tener el uso de las redes sociales, así como su desconocimiento de la religión explicaría cómo acabaron asumiendo la doctrina.

Asimismo, y pese a que es necesario seguir profundizando en el tema, se han podido discernir una serie de técnicas de manipulación que ayudaron a reformular el pensamiento de las mujeres de la muestra. No obstante, estas parecen no estar dirigidas y responden más a impulsos que a una estrategia clara de adoctrinamiento.

Por último, es necesario señalar que cualquier estrategia en el ámbito de la prevención debería tener en cuenta estas vulnerabilidades para poder atajar el problema y, siendo prioritario para frenar este proceso el refuerzo a las familias para entender y poder tratar la situación por la que están atravesando sus hijas. También son necesarias medidas dirigidas a recuperar la confianza en las instituciones y crear capital social. Una forma de crear conciencia es dar voz a las que han atravesado este proceso.

\section{Bibliografía}

BaKKer, EdWin y LeEde, Seran. 2015. «European Female jihadist in Syria: Exploring and Under- Researched Topic», ICCT Background Note, International Centre for Counter-Terrorism The Hague: 15

BENNETT, W. L. 2003. "Communicating global activism», Information, Communication \& Society, 6 (2):143-168. https://doi.org/10.1080/136 $9118032000093860 \mathrm{a}$

BLoom, Mia. 2011. Bombshell: Women and terrorism, Philadelphia: University of Pennsylvania Press.

CoOK, Joana y Gina Vale. 2018. «From Daesh to 'Diaspora': Tracing the women and minors of Islamic State», International Centre for the Study of Radicalisation (ICSR). Consultado el 25 de enero, 2020. https://icsr.info/

Cragin, R. Kim. y Sara. A. Daly. 2009. Women as terrorist: mothers, recruiters, and martyrs, Santa Barbara, Calif: Praeger Security International.

Cunningham, Karla. 2003. "Cross-Regional trends in female jihadist», Studies in Conflict \& Terrorism, 26(3): 171-195. https://doi. org/10.1080/10576100390211419 
DAVIS, JESSICA. 2017. Women in modern terrorism: from liberation wars to global jihad and the Islamic State, Maryland: Rowman \& Littlefiel.

De la Corte, Luis. 2006. La lógica del terrorismo, Madrid: Alianza Editorial.

— 2015. «Enclaves yihadistas? Un estudio sobre la presencia y el riesgo extremistas en Ceuta y Melilla», Revista de Estudios en Seguridad Internacional, 1 (2): 1-34, http://dx.doi.org/10.18847/1.2.1

— «Detenen tres presumptes jihadistes a Barcelona i Granollers». A la carta TV3, 28 de noviembre. https://www.ccma.cat/tv3/alacarta/

EsCRIVÁ, ÁNgELES. 2018, «Las semillas del mal de Tomasa ...y el destino final de los 240 españoles que se incorporaron al IS». El Mundo. 18 de febrero. https://www.elmundo.es/cronica/2018/02/18/5a87426bca4 7414b398b4680.html

García-Calvo, Carola. 2017. "No hay vida sin yihad y no hay yihad sin hégira: la movilización yihadista de mujeres en España, 2014-2016», Real Instituto Elcano. Consultado el 20 de febrero, 2020 http://www. realinstitutoelcano.org/

Gustafsson, Linus y RanstorP, Magnus. 2017. «Swedish Foreign Fighters in Syria and Iraq: an analysis of open-source intelligence and statistical data». Swedish Defence University. Consultado el 5 de enero, 2020. http://www.divaportal.org/smash/record.jsf?pid=diva2\%3A1110355\&dswid $=8515$

Hernández, Agustín et al. 2018. Barrios vulnerables de las grandes ciudades españolas, 1991, 2001, 2011, Madrid: Instituto Juan de Herrera (IJH)

Horgan, JoHn. 2005. The psychology of terrorism, London: Routledge.

Hoyle, Carolyn, AleXandra Bradford y Ross Frenett. 2015. «Becoming Mulan? Female Western Migrants to ISIS», Institute for strategic Dialogue. Consultado el 3 de febrero, 2019 https://www.isdglobal.org/ wpcontent/uploads/2016/02/ISDJ2969_Becoming_Mulan_01.15_WEB. pdf

Instituto Nacional de Estadística (INE). 2017. «Abandono educativo temprano de la población de 18 a 24 años por CCAA y periodo", Consultado el 15 de febrero, 2019. http://www.ine.es/jaxi/Datos. htm?path=/t00/ICV/dim4/10/\&file=41401.px

JORDÁN, JAVIER. 2009. «Procesos de radicalización yihadista en España. Análisis sociopolítico en tres niveles», Revista de Psicología Social, 24 (2): 197-216.

LAHOUd, Nelly. 2018. «Empowerment or Subjugation: An Analysis of ISIL's Gender Messaging», United Nations Entity for Gender Equality and the Empowerment of Women (UN Women), Consultado 13 
de marzo, 2020. https://arabstates.unwomen.org/en/digital-library/ publications/2018/6/empowerment-or-subjugation\#view

McCauley, Clark y Sophia Moskalenko. 2011. Friction: How radicalization happens to them and us, Oxford, UK: Oxford University Press.

Memoria elevada al Gobierno de su Majestad. 2016. Madrid: Ministerio de Justicia, Consultado 20 de enero, 2020. https://www.abogacia.es/ wp-content/uploads/2016/09/MEMFIS16-1.pdf

Moubayed, Sami. 2016. Bajo la bandera del terror. Un viaje a las entradas de Dáesh, Barcelona: Ediciones Península.

O’Rourke, Lindsey. 2009. "What's Especial about Female Suicide Terrorism?». Security Studies, 18 (4):681-718. https://doi. org/10.1080/09636410903369084

Peresin, Anita y Alberto Cervone. 2015. «The Western Muhajirat of ISIS», Studies in Conflict \& Terrorism 38 (7): 495-509. https://doi.org/ 10.1080/1057610X.2015.1025611

Pretus, Clara, Nafees Hamid, Hammad Sheikh, Jeremy Ginges, Adolf ToBEÑA...SCOTT ATRAN. 2018. "Neural and behavioural correlates of sacred values and vulnerability to violent extremism», Frontiers in Psychology 9 (2462):12. https://doi.org/10.3389/fpsyg.2018.02462

Q. A. 2018. «Ceuta, la única autonomía donde dejan la escuela más mujeres que hombres», El Faro de Ceuta, 14 de enero. https://elfarodeceuta.es/abandono-escolar-mujeres-femenino/

RAFIQ, HARAS. y Nikita Malik. 2015. «Caliphettes. Las mujeres, objeto y sujeto de la llamada de Daesh», Quilliam. https://beatrizbecerra.eu/ wp-content/uploads/2016/09/Caliphettes-libro1.pdf

Requena, FÉLIX. 2008. «Redes sociales y sociedad civil». Colección Monografías 256:200.

Rontomé, Carlos. 2015. «Secularización y radicalización de la población joven de Ceuta». Consultado 27 de enero, 2020. http:// www.academia.edu/30139603/SECULARIZACI\%C3\%93N_Y RADICALIZACI\%C3\%93N_DE_LA_POBLACI\%C3\%93N_JOVEN_ DE_CEUTA

SAGEMAN, MARC. 2004. Understanding terror networks, Philadelphia: University of Pennsylvania Press.

SAíz-PARdo, Melchor. 2016. «La historia de la veinteañera española que enviudó del terrorista más sádico», Diario Sur, 29 de diciembre. https:/www.diariosur.es/nacional/201612/29/historia-veinteaneraespanola-enviudo-20161229023044-rc.html

SJOBER, LAURA. 2018. "Jihadi brides and female vounteers: reading the Islamic State's war to see gender and agency in conflict dynamics». Conflict and management and peace science 35 (3): 296-311. 
Saltman, Erin Marie y Melanie Smith. 2015. «Till Martyrdom do us part, Gender and the ISIS Phenomenon», Institute for Strategic Dialogue, Consultado el 7 de marzo, 2020. https://www.isdglobal.org/isd-publications/till-martyrdom-do-us-part-gender-and-the-isis-phenomenon/

Schmid, AleX y Albert Jongman .2005. Political terrorism: a new guide for actors, authors, concepts, data bases, theories and literature, Routledge: 720.

SNOW, DAVID y RoBERT BENFORD. 1992. Master frames and cycles of protest. En A. Morris y C. McClurg Mueller (Eds.), Frontiers in Social Movement Theory, New Haven: Yale University Press.

SPECKHARD, ANNE. 2008. "The emergence of female suicide terrorists», Studies in Conflict and Terrorism 31(11): 995-1023. https://doi. org/10.1080/10576100802408121

TARRAS-WAHLBERG, Louisa. 2016. "Promises of paradise? A study on official ISIS-Propaganda Targeting Women», Swedish Defence University, Consultado el 1 de marzo, 2020, http://fhs.divaportal.org/smash/ record.jsf?pid=diva2\%3A942997\&dswid=OATDFullTextWindow

Taylor, Paul. J. y Karen Jacques. 2009. Female terrorism: a review, Terrorism and Political Violence 21 (3):299-512. https://doi. org/10.1080/09546550902984042

Torres-Marín, Jorge, Gines Navarro-Carrillo, Marcos Dono y HumBerto Manuel TrujILlo. 2017. «Radicalización ideológico-política y terrorismo: un enfoque psicosocial», Escritos de Psicología, 10 (2): 134-146. http://dx.doi.org/10.5231/psy.writ.2017.19042

Trujillo, Humberto Manuel. 2009. «Hacia una mejor comprensión psicológica del terrorismo: reclutamiento, ideología y violencia». Revista de Psicología Social 24:163-181. https://doi. org/10.1174/021347409788041426

Trujillo Humberto Manuel, Alonso Ferrán, José Miguel Cuevas y Manuel Moyano. (2018), "Manuel Evidencias empíricas de manipulación y abuso psicológico en el proceso de adoctrinamiento y radicalización yihadista inducida», Revista de estudios Sociales, 66: 42-54, https://doi.org/10.7440/res66.2018.05

«UNEMPLOYMENT RATES IN THE UE REGIONS RANGED FROM 1.7\% to 29.1\%». 2018. Eurostat, Consultado 13 de enero, 2020, https://ec.europa. eu/eurostat/documents/2995521/8830865/1-26042018-AP-EN.pdf/ bb8ac3b7-3606-47ef-b7ed-aadc4d1e2aae

UNIÓN DE COMUNIDADES IsLÁMICAS DE EsPaÑa (UCIDE).2018. «Estudio demográfico de la población musulmana», Observatorio Andalucí. Consultado 17 de enero, 2020, http://observatorio.hispanomuslim.es/ estademograf.pdf

Victor, Barbara. 2004. Army of Roses. Inside the World of Palestinian Women Suicide Bombers, London: Constable \& Robinson. 


\section{JURISPRUDENCIA}

Audiencia Nacional. 2016. Juzgado Central de Instrucción nº1, Auto de procesamiento, sumario 3/2016, 29 de enero, Madrid

Audiencia Nacional. 2018. Juzgado de Instrucción nº1, Escrito de Acusación del fiscal, sumario 5/2016, 21 de mayo, Madrid

Audiencia Nacional. 2016. Juzgado de Instrucción n ${ }^{\circ} 1$, Sumario 5/2016, 16 de febrero, Madrid

Audiencia Nacional. 2016. Juzgado de Instrucción nº 6, Auto de procesamiento, sumario 3/2016, 26 de mayo, Madrid

Audiencia Nacional. 2017. Juzgado de Instrucción $\mathrm{n}^{\circ} 2$, Sentencia 11/2017, 17 de marzo, Madrid

Audiencia Nacional. 2017. Juzgado de Instrucción n², Sentencia 5/17, 28 de febrero, Madrid

Audiencia Nacional. 2018, Juzgado de Instrucción $n^{\circ}$ 5, Sentencia 33/2018, 25 de septiembre, Madrid 